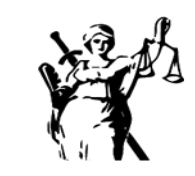

JUSTICIA

ISSN impreso 0124-7441
ISSN digital 2590-4566

\title{
Path dependence en el desarrollo histórico-institucional, político- administrativo y sectorial en Colombia (1960-2020)
}

\author{
Evidence of path dependence in the historical-institutional \\ development of the agricultural and industrial sectors in Colombia \\ (1960-2020).
}

\author{
Rafael Vergara Varela \\ Universidad del Valle, Colombia \\ rafael.vergara@correounivalle.edu.co
}

Recibido: 12 de diciembre de 2019 / Aceptado: 11 de marzo de 2020

https://doi.org/10.17081/just.25.37.3673

\section{Resumen}

El presente artículo estudia y analiza el desarrollo institucional colombiano en medio de la periodización determinada. Por tanto, este análisis se armoniza bajo el contexto teórico del institucionalismo histórico herramienta esencial en las ciencias sociales. En medio de esta temporalidad se presentan dos coyunturas entre (1958-1989) y (1990-2020) que permiten evidenciar el cambio institucional en el país. Asi bajo este periodo de tiempo se aplica el modelo teórico al presente estudio y de paso se evidencia el supuesto del path dependence o path dependency en Colombia.

Palabras clave: institucionalismo histórico, políticas públicas sectoriales, administración pública, path dependence, Colombia.

\begin{abstract}
This article studies and analyzes the Colombian institutional development in the middle of the determined periodization. Therefore, this analysis is harmonized under the theoretical context of historical institutionalism. In the middle of this periodization, there are two junctures between (1958-1989) and (1990-2020) that show the institutional evolution in the country. Thus, under this period of time, the theoretical model is applied to the present study and, in passing, the assumption of the path dependency or path dependence in Colombia is evidenced.
\end{abstract}

KEYWORDS: historical institutionalism, sector public policies, public administration, path dependence, Colombia.

\section{Como citar:}

Vergara Varela, R. (2020). Path dependence en el desarrollo histórico-institucional, político-administrativo y sectorial en Colombia (1960-2020). Justicia, 25(37), 85-98. https://doi.org/10.17081/just.25.37.3673 


\section{INTRODUCCIÓN}

En el presente artículo se socializan los resultados de una investigación doctoral sobre el desarrollo institucional colombiano. En razón a ello, este documento busca exponer de manera teórica y aplicada un análisis transdisciplinar centrado a las instituciones en Colombia teniendo en cuenta la cronología. Bajo esta orientación se expone también el paradigma del neo institucionalismo como marco de estudio en las ciencias sociales para explicar sus principales enfoques en especial el institucionalismo histórico.

Ahora, en relación al objeto de trabajo como es el institucionalismo histórico se explica pedagógicamente su modelo de estudio, análisis, abordaje y aplicación. Así se expone el supuesto de path dependence o path dependency como elemento central del modelo. En la segunda parte del documento se presentan los resultados en una investigación aplicada en torno a la temática. En último tercio están las conclusiones.

Finalmente, el objetivo primordial de este artículo busca explicarles al lector académico y al público en general las posibilidades de trabajo como análisis en la dinámica del institucionalismo histórico, siendo este enfoque una herramienta relevante en las ciencias sociales. Por lo tanto, se expone a continuación la discusión metodológica de una manera pedagógica, práctica y aplicada.

\section{Paradigma del neo institucionalismo y su enfoque del institucionalismo histórico.}

Para (North, 2010) en un análisis holístico y sistémico, define que las instituciones están correlacionadas con los mercados, con el sistema jurídico como administrativo, con la estructura social y el contexto histórico dado que los cambios políticos son influyentes, ya que actúan para acelerar o frenar el sistema económico. Este autor afirma también que las instituciones proveen la estructura básica en el cual los seres humanos han creado orden y reducido la incertidumbre del cambio en la historia. En si las instituciones permiten a los agentes eliminar la incertidumbre mediante la experiencia ya concretada en estas, sirviendo de base sólida para formar y mantener procesos de estabilidad estructural (North, 1994).

Así pues, los estudios e investigaciones sobre el cambio, la continuidad y evolución en el desarrollo de los países e instituciones han sido abordados desde diferentes contextos teóricos teniendo como objeto de análisis a: los mercados, las organizaciones, los sujetos, las colectividades y la historia. Desde el marco cognitivo del neo institucionalismo se han analizado diversas perspectivas en las ciencias sociales siendo orientadas a través de diferentes temáticas conocidas como: el enfoque de elección racional, la elección pública (public choice) el institucionalismo organizacional, la escuela sociológica, y el enfoque del institucionalismo histórico.

Como tal, y de acuerdo con Raymundo García (2013: 6) "el neo institucionalismo es un instrumento teórico que ayuda a explicar la creación, desarrollo, funcionamiento, y cambio o extinción de las instituciones". Desde las diversas escuelas de pensamiento, los neo-institucionalistas ven a estos enfoques cognitivos y referenciales como el factor de incidencia en los sujetos, sus comportamientos, o como el centro del estudio. Por ejemplo, el enfoque de elección racional (rational choice) expone la toma de decisiones o elección de los individuos como variable económica principal en el desarrollo institucional mientras que el institucionalismo sociológico da cuenta de un macro nivel de variables y/o categorías sociales que en ocasiones puede incluir tanto las de elección racional como las del enfoque histórico (Murillo, González y García, 2010: 89).

Asimismo, este último enfoque se centra en el papel de las instituciones y su dinámica económica 
también en los comportamientos, coyunturas y evolución prestando atención a los antecedentes y a los resultados a posteriori en un marco analítico de larga duración donde la variable principal es la historia (Cita 11, En: Basabe, 2007: 180).

La incidencia de las instituciones en los comportamientos y elecciones de los agentes, desde la elección racional y el institucionalismo histórico más que diferenciarse como expresa Sánchez de dios (2004: 98), lo que hacen es complementarse, ya que si bien los racionalistas exponen que las instituciones importan como factores del contexto estratégico de los actores, y para los históricos las instituciones tienen un papel más que relevante al cuestionar la capacidad de los actores para diseñar y llevar a cabo condiciones óptimas debido a su dependencia con el pasado, o los antecedentes que presentan para decidir sus comportamientos. Empero, pese a la diferencia en conceptualizaciones, en ultimas ambos se armonizan pues se puede considerar que las instituciones son factores del contexto enmarcadas históricamente como antecedentes, que permiten a los agentes elegir con base en ideas ya preestablecidas, disminuyendo con ello los costos de transacción y niveles de incertidumbre.

Como tal, lo anterior forma parte de los efectos de los mecanismos con base en la teoría de los rendimientos crecientes que Pierson (2000: 254; En: Ibíd: 100 - 101) aplica al desarrollo institucional; tales como el mecanismo de elevados costes de establecimiento, que incentiva a los actores a identificarse y ajustar comportamientos con base en opciones establecidas asi; el efecto aprendizaje, en el cual el conocimiento obtenido incentiva el uso continuo del sistema; el efecto coordinación, donde los agentes se adaptan a nuevas situaciones según los beneficios obtenidos; y el efecto adaptación de expectativas, en el que los beneficios previstos de seguir una pauta conlleva a reforzarla.

El centrar la mirada en las instituciones como punto de partida de los comportamientos se concibe para incidir en la disminución de costos de transacción en el ámbito de las decisiones sociales y la solución de problemáticas, presentando implícitamente relaciones de causalidad entre las instituciones y los agentes que condicionan tanto la acción, como las estrategias y los resultados (North, 1990; Diermeier y Krehbiel, 2003, En: Marenco, 2006: 54 - 55).

Por ello, el institucionalismo histórico además de plantear la relación entre agentes e instituciones, definiendo los limites en que los actores adoptan sus estrategias y líneas de acción; permite conocer también la evolución de los participantes en medio de conflictos instaurados en el tiempo (Sánchez de dios, 2004: 97). Conflictos entendidos como escenarios temporales, puntos de cambio histórico y coyunturas que permiten explicar las dependencias del pasado, (path dependence o path dependency) cuestionando incluso la racionalidad para elegir, estando limitado a soluciones ya preestablecidas dadas las secuencias de su evolución institucional.

Ahora, desde el ámbito de las políticas sociales y la gestión pública, como expresa Farfán (2007: 87) el institucionalismo histórico proporciona un enfoque u herramienta académica para estudiar las políticas públicas transdisciplinariamente permitiendo además un dialogo de saberes integrando la historia, la administración, la economía, el derecho, la ciencia política, la sociología e incluso la cliometría en el análisis institucional a un objeto de estudio. En sí, el planteamiento central es la evolución institucional (Saavedra, 2016: 91) considerando las instituciones como construcciones dadas en el tiempo, que se reproducen y cambian, modificando e introduciendo las reglas de juego, por lo cual su metodología es interpretativa y a largo plazo. (Krasner, et al, 1984; Pierson, 2000, 2004; Thelen, 1999, 2006; Thelen y Streck, 2005; En: Saavedra, 2016: $84-85)$.

Por esto, al no ser un enfoque cortoplacista, su estudio, análisis, abordaje y aplicación investigativa 
se concentra en los patrones institucionales en largo duración donde la historia como variable principal y explicativa del desarrollo se acompaña de argumentos presentes e identificables de orden metodológico como son: los puntos de equilibrio, las secuencias, las coyunturas críticas, su evolución y su tendencia (Vergara, 2019).

Por ello, el institucionalismo histórico define a las instituciones como el legado de procesos históricos donde existe una sincronización y temporalidad (Trigo, 2016: 230), donde también puede existir estabilidad o fluidez con procesos de apertura, contingencia y agencia sobre los cierres en el desarrollo institucional (Saavedra, 2016: 83). Con base en Hall y Taylor (1996: 938; En: Trigo, Óp. cit: 229) este enfoque se caracteriza por lo siguiente: Primero, su conceptualización sobre la relación entre las instituciones.

Segundo, la manera en cómo destaca las asimetrías de poder en el desarrollo de las instituciones. Tercero, su visión del desarrollo institucional donde aparece el concepto de path dependence o path dependency. Y cuarto, su preocupación por el análisis integrando la contribución de otras disciplinas académicas que permiten explicar un resultado.

Finalmente, el considerar el componente histórico para entender las dinámicas políticas, jurídicas, sociales, administrativas y demás, define de entrada una explicación basada en la dimensión temporal de los procesos según lo expone Pierson (2004: 2; En: Olano, 2014: 7), razón por la cual se debe al utilizar este enfoque definir los periodos de análisis en los que se observara la crisis, cambio o continuidad del objeto de estudio. Dentro de este contexto de análisis histórico los periodos, los puntos críticos o de rupturas juegan un rol importante, asi aparece en escena el "path dependence" o "sendero de la dependencia" como el contexto definido por las decisiones iníciales y que permite comprender bajo un escenario de larga duración a las instituciones en su forma actual (Liebowitz, \& Margolis, 1995).

\section{El supuesto teórico del path dependence o path dependency.}

Básicamente, el argumento teórico del institucionalismo histórico juega un papel relevante en el análisis investigativo porque se centra en la capacidad de analizar escenarios desde una perspectiva de largo plazo o (larga duración histórica), al ser este su centro de análisis. Por ello, emerge el concepto de la dependencia del pasado o "path dependence", en el cual se describe una rutina institucional que se reproduce en el tiempo, lo que significa que siempre se conservan sus rasgos fundamentales.

Por ende, esta dependencia del sendero, o dependencia del pasado describe algo así como la existencia de movimientos cumulativos que se repiten en la historia de las sociedades. Lo que implica también desde otra lectura que la herencia institucional y política, pesan y se reproducen continuamente en la historia (David, 2001; Valdivieso, 2008; Boussaguet, 2016; Piña López, Medina Zárraga, \& Curiel Gutiérrez, 2017)

Dicho de otra forma, el interés y aporte del institucionalismo histórico por el estudio de las instituciones en el largo plazo conlleva a observar en estas los procesos y factores que han incidido en ellas, por lo cual se debe vislumbrar si existen secuencias distintivas que hagan que los actores implicados continúen rutas establecidas (Pierson, 2000: 264; En: Farfán, 2007: 92). La continuidad en dichos caminos ya señalados da cuenta de la existencia de unas trayectorias de camino, o de path dependence. De igual manera un cambio en las secuencias y la estabilidad presenta y expone la existencia e inicio de coyunturas críticas, o critical junctures.

Tanto las coyunturas como las trayectorias definidas son parte de los modelos de análisis y perspectivas sobre crisis, continuidad y cambio en el enfoque del institucionalismo histórico, como lo expresa Trigo 
(2016: 231 - 235), los cuales responden a la estabilidad, reproducción y modificación de instituciones, que no tienen siempre que reemplazar lo anterior, sino presentar la existencia de variaciones en patrones en el contexto político y social más no su cambio inmediato y total.

El path dependence o la dependencia de camino es un término que permite indicar la relación entre las decisiones actuales y las pasadas, en si el condicionamiento generado desde el pasado hacia el futuro (Marín - García, Perello y García, s.f.: 37 - 38). Desde el institucionalismo histórico, esta teoría presenta la probabilidad de transitar por estadios y resultados similares a los antecedentes de un proceso, de acuerdo con el impacto que estos hayan generado en los agentes y el contexto.

De acuerdo con Marín-García et al (s.f.; 38) como condiciones de esta teoría se encuentran la existencia de costes de cambio elevados tras la toma de decisiones, y la importancia y consecuencias que presentan las decisiones iniciales. Así, no se realizarán grandes cambios en las trayectorias institucionales, dado que la incidencia de la historia en esta, de los factores, el contexto y los actores; ha marcado una serie de rutas de acción que determinan los comportamientos, disminuyendo los niveles de incertidumbre en los procesos de elección a posteriori, presentando relaciones causales determinadas entre las opciones a elegir y los posibles resultados.

El disminuir la incertidumbre no responde más que al reforzamiento mutuo en el ajuste y la selección competitiva de trayectorias que se basan en los resultados existentes (Pierson, 2000: 812; En: Sánchez de dios, 2004: 96). Es importante destacar que el reforzamiento se debe a la incidencia de ciertos agentes, pues en el desarrollo del juego sociopolítico e histórico, las luchas por el poder en los diferentes escenarios han marcado los caminos, las ideas y las conceptualizaciones que sirven de guía en la toma de decisiones por parte de los actores.

Empero, la existencia de ciclos históricos repetitivos en el ámbito institucional no solo da cuenta de la dependencia al pasado, también puede significar que en inicio la estructura institucional respondió a ciertos factores, presentando la génesis de las instituciones o su branching poitns (punto de partida). Como expone Pierson (2004; En: Marenco, 2006: 56) en los procesos de path dependence no solo existen temporalidades de larga duración, inmersas en estas cohabitan periodos cortoplacistas donde las instituciones se originan o pueden variar, modificando su continuidad o evolucionando sin alejarse de su camino guía, así esta pueden responder tanto a factores funcionales como ser producto de eventos discretos o consecuencias no previstas estratégicamente, generadas por la inmediatez del momento, pero cuyo impacto a futuro puede continuar definiendo la existencia del ciclo histórico.

El hecho de que las instituciones sigan un path o puedan variar debido a una coyuntura hace que en el análisis desde el institucionalismo histórico se prevean los momentos cruciales en la formación y desarrollo de las instituciones, como de igual manera, los momentos de adaptación y evolución al contexto.

En sí, la teoría del path dependence aporta rigor al análisis institucional, a través de cuatro aspectos centrales con base en Pierson (2000b: 263; En: Sánchez de dios, 2004: 99 - 100): primero, un principio de equilibrio múltiple, en el que un grupo de condiciones base pueden dar múltiples soluciones posibles; segundo, un principio de contingencia, donde los aspectos y acontecimientos irrelevantes cobran importancia en el desarrollo histórico institucional en el largo plazo; tercero, una temporalidad y secuencia, en las cuales las primeras fases de desarrollo son determinantes en la historia; y cuarto, un principio de inercia, donde el retroceso conlleva a la búsqueda y generación de equilibrios. 


\section{Aplicación del institucionalismo histórico al contexto colombiano (1958-2020).}

A manera de síntesis, el marco teórico del institucionalismo histórico estudia el contexto, el comportamiento, la secuencia y la transformación de las instituciones. Además se concentra en el análisis panorámico o de larga duración histórica. Igualmente estudia la relación entre agentes e instituciones por intermedio de conflictos instaurados en el tiempo. Es decir, conflictos determinados como escenarios de cambio que permiten explicar las dependencias del pasado. En últimas, estas instituciones se reproducen cambiando las reglas del juego en el tiempo. Por ello, la historia es la variable dependiente y explicativa del desarrollo institucional (David, 1994: Alexander, 2001: Sánchez de Dios, 2004: Saavedra, 2016).

Este modelo analítico-teórico del institucionalismo histórico consta de las siguientes cuatro etapas para su estudio, comprensión, análisis, abordaje y aplicación investigativa, asi: Uno, subperiodos de puntos de partida, puntos críticos o generación de nuevos puntos de equilibrio. Dos, coyunturas (temporales o estructurales). Tres, secuencia general del proceso histórico. Y cuatro, tendencia y/o pronóstico del objeto de estudio (Saavedra, 2016; Vergara, 2019). Esquemáticamente este modelo se representa asi (ver figura uno):

Figura 1. Modelo esquemático del neo institucionalismo histórico y sus etapas de análisis

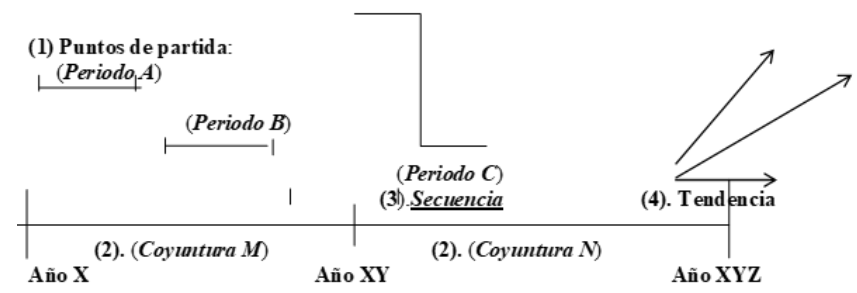

Fuente: Elaboración propia con base en el marco del institucionalismo histórico, 2019.

A continuación se presenta como ejemplo el producto de una investigación que responde al contexto histórico del desarrollo institucional en Colombia (Vergara, 2019). En este estudio se aplica el marco del institucionalismo histórico. El período investigado corresponde a dos escenarios coyunturales y estructurales de los últimos 60 años (ver figura 2).

Figura 2. Escenarios coyunturales y estructurales económicos en Colombia (1958-2020

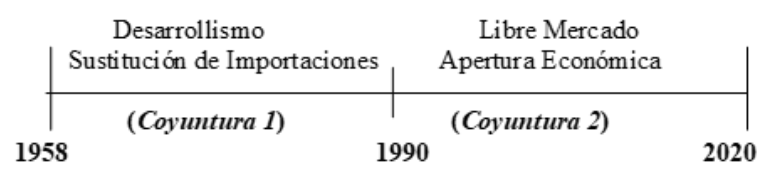

Fuente: Elaboración Propia, 2019

En la figura dos se estudia y analiza un escenario de larga duración en la historia reciente de Colombia, (donde hay dos modelos institucionales en el desarrollo en el país). Es decir, Colombia ha trasegado en dos estructuras económicas claramente establecidas y definidas: La primera coyuntura va desde 1958 hasta 1989 donde su modelo institucional se define en el marco de una economía cerrada y proteccionista bajo un contexto normativo de sustitución de importaciones cuyo fin buscaba fortalecer los sectores productivos para el desarrollo colombiano. Esta política institucional buscó proteger y mejorar la producción nacional 
en la generación de bienes de consumo y en la producción de bienes intermedios. Empero, la excepción a la norma aplicaba solo para bienes de capital y para algunos bienes intermedios o insumos tecnológicos necesarios para la producción nacional (Villar \& Esguerra, 2005). Posteriormente la segunda coyuntura inicia desde 1990 bajo el influjo de la apertura económica global. Al respecto, el modelo del neoliberalismo es transferido al país por el diseño institucional y normativo de las políticas de mercado definidas por los organismos internacionales como: ONU, Fondo Monetario Internacional, Banco Mundial, OEA, Banco Interamericano de desarrollo etc. (Rendón, 1998; Vergara \& Rodríguez, 2015: Baena \& Fernández, 2016).

Así pues, recreando y armonizando la teoría del institucionalismo histórico con la realidad colombiana se encuentra también que al interior de este estudio se hallaron cuatro subperiodos de que corresponden a puntos críticos de cambio institucional. Estos subperiodos (ver figura 3) obedecen y se articulan a puntos de partida delimitados cronológicamente al interior de las dos coyunturas históricas descritas en la página anterior, así: Uno, (1958-1974) el periodo del "Frente Nacional". Dos, (1975-1989) denominado "transición institucional". Tres, (1990-2001) el escenario de la "apertura económica". Y cuatro (2002-2020) establecido como la "reprimarización de la economía" (Vergara, 2019).

Figura 3. Evolución económica e institucional en Colombia (1958-2020)

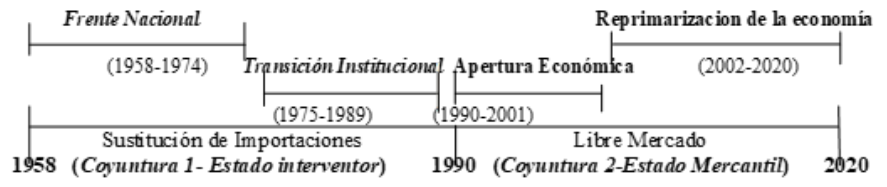

Fuente: Elaboración Propia, 2019

\section{RESULTADOS}

Haciendo el análisis de 61 años se encuentran dos coyunturas como las descritas en la figura tres, al interior de cada coyuntura se definieron desarrollos institucionales propios en el periodo. Ahora bien, en este estudio la primera coyuntura institucional en Colombia entre 1958 y 1989 (se conoció como desarrollismo bajo la política de un Estado interventor) cuyo modelo buscó impulsar los sectores productivos del país. Por ejemplo: el modelo proteccionista y la herramienta normativa de la sustitución de importaciones permitieron generar la industria nacional de bienes de consumo y en algunos rangos la producción de bienes intermedios. Solo, las importaciones para el sector industrial fueron de insumos tecnológicos para bienes intermedios y bienes de capital. También, el Estado interventor definió políticas para el sector agropecuario basadas en subsidios de insumos, fertilizantes, semillas, agroquímicos, herramientas, maquinaria y créditos.

Asimismo, desde el contexto político se desarrolló en Colombia el retorno a la democracia bajo el esquema institucional del Frente Nacional entre 1958 y 1974 donde cada cuatro años hubo una alternancia de poder entre los partidos liberal y conservador (Fajardo, 2016). Bajo esta periodización también se impulsó normativamente y se implementó la descentralización jurídico-política y administrativa hacia las regiones bajo la arquitectura institucional de la reforma constitucional de 1968. (Balcázar et al, 1998; López, 1988; Perdomo, 2004; Paredes \& Díaz, 2007).

Al intermedio de esta primera coyuntura se presentaba también un segundo punto crítico o punto de cambio entre 1975 y 1989 que mostraba una transición institucional para atenuar gradualmente el protec- 
cionismo estatal y la sustitución de importaciones impulsado por el paradigma de libre comercio. También en este periodo se hacen evidentes los efectos de la crisis internacional de los precios del petróleo (1975) y de la crisis de la deuda latinoamericana de los años ochenta denominada institucionalmente como la década perdida o la crisis de la deuda. Por su parte, esta transición institucional desde el ámbito jurídico-político también se generó a posteriori por el influjo de la reforma constitucional de 1968, es decir se dio una atenuación gradual del centralismo decisorio bogotano. En consecuencia se dando una descentralización política y jurídico-administrativa hacia la autonomía de las regiones bajo la implementación de la elección popular en su orden: Senadores, Representantes, Alcaldes y Concejales. (Díaz-Alejandro, 1985; Sánchez, 2000; Maldonado et al, 2001; Gaviria, 2003).

De otro lado, en relación a la segunda coyuntura o (coyuntura dos) esta se inicia desde 1990 en adelante. A partir de este año se implementa institucionalmente un nuevo escenario de libre mercado con directriz de ajuste estructural político, económico y sectorial. La adopción e institucionalización del modelo de apertura económica se realizó en el gobierno presidencial de Cesar Gaviria Trujillo. La implementación de esta política fue inminente, brusca y radical además sin tener en cuenta los costos sociales y económicos que generaría en el país. Básicamente, este modelo institucional respondió al influjo de los organismos multilaterales como: el Banco Mundial, el Fondo Monetario Internacional, etc.

En esencia el mercado se superpone a la acción del Estado. Por ejemplo: la inversión a los sectores productivos ya no será asumido totalmente por el Estado es decir, esta nueva estructura responde a las dinámicas del mercado, el capital extranjero, y la financiación de los actores privados. Igualmente desde lo administrativo y lo jurídico como parte del ajuste estructural se originó el cambio institucional con la Constitución Política de 1991 dando respaldo normativo al nuevo modelo del Estado empresario mercantilizado (Hidron, 2001; Castaño, 2002; Nájar, 2006; Cáceres, 2013; Herrera, 2013).

Bajo este mismo influjo paradigmático, actualmente en el nuevo milenio la administración del Estado se inscribe en las retoricas del mercado internacional bajo competitividad, rentabilidad y crecimiento económico en la inversión de comodities. Este periodo se conoce como: "la reprimarización de la economía colombiana", este modelo se concentra en la exploración y explotación de recursos minero-energéticos e hidrocarburos elementos que responden a las retoricas y apuestas rentables del comercio global (González, 2011; Nova, 2014; Sarmiento \& Pérez, 2016).

Por otra parte, en complemento a este estudio, la figura cuatro se presenta la evolución de la planificación y la estrategia. Al respecto, la Ley 19 de 1958 reguló y redefinió las funciones de la administración pública y del derecho administrativo como también la planificación del desarrollo económico y la creación del Departamento Nacional de Planeación (DNP).

Figura 4. Evolución económica, organizacional e institucional (Colombia (1958-2020)

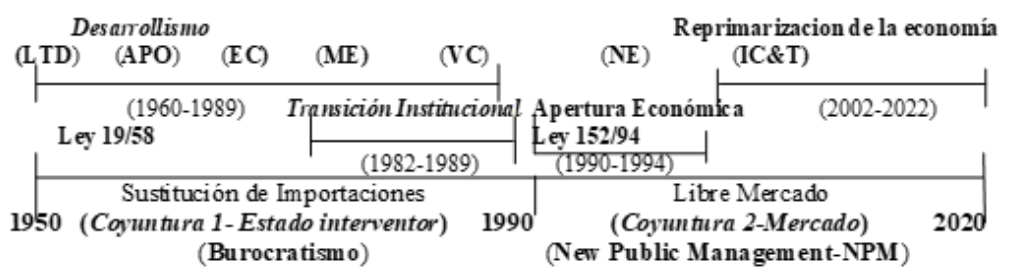

$\mathbf{L T D}=\mathrm{Liderazgo} y$ toma de decisiones, $\mathbf{A P O}=$ Administración por objetivos, $\mathbf{E} \mathbf{C}=\mathrm{E}$ strategia corporativa, $\mathbf{M E}=$ Management estratégico, VC=V entaja competitiva, $\mathbf{N E}=$ Nueva economia, IC \&T=Innovación, c \& tech

Fuente: Elaboración Propia, 2019 
En este contexto histórico-institucional los programas de gobierno presidenciales y/o planes nacionales de desarrollo en el caso colombiano se han sancionado luego de una profunda deliberación, debate y discusión por parte de actores con poder político denominados stakeholders (llámese gremios sectoriales y bancadas regionales de senadores y representantes en el Congreso de la Republica). Por ello hay que anotar que la planeación estratégica es a la administración privada como también la política pública es a la gerencia y a corporaciones públicas. Por esto los planes nacionales de desarrollo obedecen a una relación o sumatoria entre el polity y el politics lo que da como resultado el policy. En conclusión en el caso colombiano los planes nacionales de desarrollo son política pública. Esto indica que la administración presidencial gobierne por políticas públicas.

Al analizar la figura 4 se entiende entonces que la planeación aplicada en la administración pública colombiana en especial a los planes nacionales de desarrollo responde a indicadores cualitativos en relación a lineamientos de política, objetivos de política y metas. Bajo esta metodología los planes nacionales de desarrollo en Colombia después de 1958 responden al escenario organizacional de la administración por objetivos (APO) de Peter Drucker. Esto explica también una dependencia del pasado en las temáticas de la administración y el derecho público en Colombia. Además esta dinámica se ha mantenido así porque el método de la planificación estratégica en los planes de desarrollo solo ha sido sancionado y regulado en el país mediante la Ley 152 de 1994. Es decir por casi más de 40 años se ha realizado en la administración pública colombiana una aplicación metodológica de la administración por objetivos con aspectos de toma de decisiones de racionalidad limitada.

Este escenario descrito arriba se sustenta también porque la administración presidencial en Colombia es de cuatro años de corta duración (si se la compara frente a otros escenarios políticos mundiales que incluyen re-elección). Sumado a ello, la ejecución de los planes de desarrollo corresponde al tiempo que queda luego de su revisión y deliberación en el Congreso de la Republica que por lo regular el tiempo de ejecución de las programas de gobierno suele ser de más o menos tres años de la administración presidencial. En esta orientación, por lo menos los planes nacionales de desarrollo entre 1960 y 1994 no han tenido una planeación estratégica como tal porque en Colombia las políticas de Estado no existen. Es más la falta de una visión de política de Estado sumado a los intereses egoístas de los grupos de poder explica la crisis institucional y económica hoy.

Otra de las razones del por qué no se ha aplicado una planeación estratégica a los planes nacionales de desarrollo con una orientación de política de Estado obedece también porque ninguna administración presidencial en Colombia ha logrado comprometer las vigencias futuras de sus sucesores. Valga la pena plantear que desde el análisis del derecho administrativo comprometer las vigencias futuras y/o presupuestos futuros implica una apertura de una investigación de contraloría, procuraduría y fiscalía. Por este hecho en Colombia se han investigado, destituido y sancionado a funcionarios públicos. Asimismo, los planes nacionales de desarrollo en Colombia no comprometen el largo plazo. Cabe recordar además que las formas de planeación para planes de desarrollo se instalan metodológicamente como se planteó anteriormente bajo el modelo de la administración por objetivos, el cual responde también desde el punto de vista del presupuesto y financiación de proyectos sujeto a las metodologías de los organismos multilaterales y de la banca internacional siendo este un modelo que hegemónicamente se conoce como bottom-up.

La planeación estratégica con visión a futuro ha aparecido tímidamente después del año 1998 en los planes nacionales de desarrollo en Colombia apuntando a temas como: la competitividad, las ventajas comparativas, la ciencia, la tecnología y la innovación. En este aspecto los planes de desarrollo de los últimos 20 años se han orientado con visión de Estado en torno a la planeación, la exploración y la explotación de los recursos naturales-minero-energéticos e hidrocarburos como en el caso del petróleo, el carbón y 
el gas, al ser estos bienes transables y competitivos los denominados comodities se tasan por sus altos precios en el mercado internacional.

Ahora se presenta otro objeto de análisis bajo el marco del institucionalismo histórico, se analizará el grafico uno para verificar la posible presencia de path dependence. Frente a esta noción se presenta un caso de manera ilustrativa para hacer más comprensible y aplicable el concepto. Por ejemplo: en relación al avance tecnológico, el teclado convencional (QWERTY) aparece desde el origen de la máquina de escribir en 1714. Este teclado también responde a un pasado histórico que se ha configurado en máquinas de escribir manuales y eléctricas, computadores, agendas electrónicas, celulares inteligentes (smartphones), tablets, reproductores de sonido como video y televisores (Smart tv). Esto significa que a través del tiempo a medida que ha evolucionado la tecnología también se ha mantenido intacto el teclado clásico convencional (QWERTY). Es decir se ha conservado culturalmente en el tiempo y no ha tenido modificación alguna hasta hoy en plena cuarta revolución industrial. Por lo tanto, este arquetipo de letras enmarcado en un teclado bajo esquema (QWERTY) se delinea desde el pasado a hoy en una senda de path dependence.

Grafico 1. Aplicación del path dependence sector agropecuario e industrial en Colombia (1958-2018)

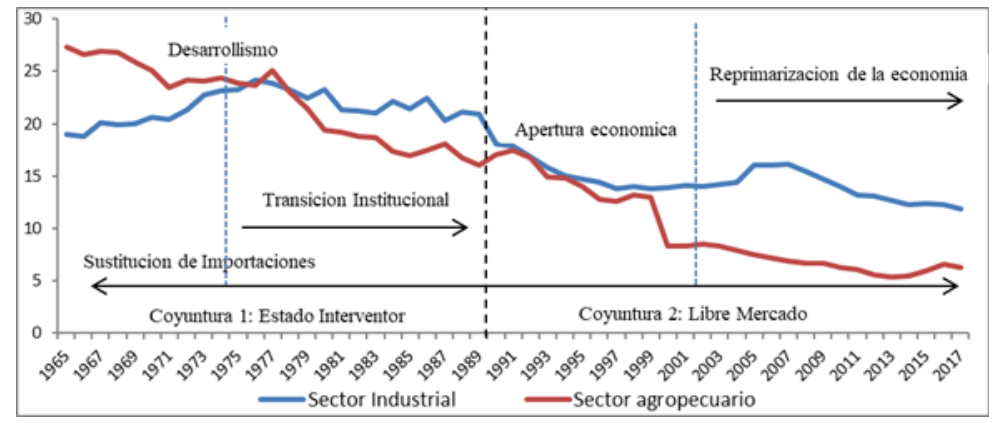

Fuente: Elaboración propia con base en datos estadísticos del Banco Mundial, 2019.

Teniendo claro el ejemplo anterior, se aplicara el marco del institucionalismo histórico a dos sectores productivos en Colombia como son: el sector agropecuario y el sector industrial (ver gráfico arriba). En el gráfico uno tenemos dos coyunturas: Una (1960-1989) en el contexto del Estado interventor bajo sustitución de importaciones y promoción de las exportaciones. Dos, a partir de 1990 se institucionaliza el modelo de libre mercado y en el primer cuatrenio de la década de los años noventa se instauran las reformas políticas, económicas y sectoriales. Como se puede notar a partir de 1974 los sectores agropecuario e industrial empiezan a perder dinamismo en la economía colombiana. Cabe también recordar que entre 1974 y 1989 , los gobiernos presidenciales generaron reglas del juego de transición institucional de orden económico y político. Entre estas se encuentran las licencias globales de importación, la importación de bienes de capital, la descentralización territorial, la elección popular de alcaldes y gobernadores, etc.

Básicamente en el periodo (1974-1989) se presenta un decrecimiento de los dos sectores más productivos del país. En el caso del sector industrial los factores obedecen a un rezago tecnológico que responde a una dependencia en la importación de tecnología como también a una escasa formación de capital humano y a una no decidida acción política en función del cambio técnico que Colombia requería para ese momento. Es decir, la gestión pública de la planificación del desarrollo no tiene los efectos esperados entre 1974 y 
1989 dado que se esperaba que el sector industrial como sector líder jalonara el desarrollo del sector agropecuario. Además la coyuntura está mostrando que el sector industrial empieza a decaer y por ende este declive no apalanca el desarrollo del sector agropecuario. Por lo tanto, el sector agropecuario es un sector afectado en la planeación del desarrollo.

Por otra parte, a partir del año 1990 se da un giro en el modelo económico hacia el contexto de libre mercado. Desde la literatura económica se planteó que el nuevo modelo de apertura económica diversificaría la economía colombiana generando un crecimiento de sus sectores productivos. Sin embargo, la realidad reflejó todo lo contrario (Fierro, 2015). Es decir, a pesar de que en 1990 hubo un cambio institucional: político, económico y sectorial, se esperaba una mejoría de los sectores productivos. Pero, la trayectoria histórica de los sectores agropecuario e industrial no se modifico es más con la implementación del nuevo modelo de la apertura económica se acentuó más rápidamente la desaceleración de ambos sectores. En conclusión se revelan dos escenarios entre (1958-1989) y (1990-2019) donde en un análisis de larga duración hay un declive sectorial a partir de 1974. Por lo tanto, desde 1990 el nuevo modelo o paradigma económico del mercado no transformo, ni modifico la tendencia previa de (1958-1989) lo cual demuestra una continuidad en la caída de la tendencia sectorial definiendo por demás en un análisis panorámico, una trayectoria de camino decreciente que responde naturalmente al supuesto del neo institucionalismo histórico del path dependency o path dependence.

En cuanto a su pronóstico, cabe agregar que la tendencia de los sectores agropecuario e industrial de no modificarse en el corto plazo afectará (en los próximos 10 años) de manera negativa al producto interno bruto del país restándole dinámica a la economía colombiana En síntesis, la gestión de la planeación del desarrollo tiene desaciertos dado que al formularse políticas para periodos de gobierno presidencial tan cortos no se tiene en cuenta un proyecto del desarrollo del país a largo plazo. Esto resalta que los planes de desarrollo fallan al no establecer criterios metodológicos de planeación estratégica y de prospectiva.

Finalmente, al investigar y contrastar lo planificado con la realidad, se encuentra que hay evidencia que al verificarla con el marco teórico del neo institucionalismo histórico se cumple el supuesto del path dependence para los sectores en medio de la cronología (1958-2014). En este sentido presente artículo responde al método de investigación propuesto.

\section{CONCLUSIONES}

El paradigma del nuevo institucionalismo es una herramienta importante desde el análisis transdisciplinar ya que permite investigar, trabajar y abordar casos de estudio complejos que requieren el análisis integral de otras disciplinas. Cabe recordar también que este marco teórico describe unos sub-enfoques que se articulan al análisis investigativo y académico como: el racional, el organizacional, public choice, el sociológico y el institucionalismo histórico.

El estudio expuesto aquí respondió a la dinámica y enfoque del institucionalismo histórico donde este marco cognitivo se utilizó para el desarrollo de un investigación doctoral (Vergara, 2019). En este artículo se socializan parte de los resultados y de los hallazgos de la tesis donde su argumento central fue probar la aplicación como la presencia y existencia del supuesto del path dependence a la planeación y a los sectores agropecuario e industrial en Colombia. Por ello, hasta la fecha de publicación de este artículo no existe en el país una tesis en pregrado o postgrado u otra publicación que hubiera estudiado e investigado este caso en el entorno de las ciencias sociales. Esta tesis del doctorado en administración fue evaluada por pares internacionales expertos en políticas públicas y gestión pública además la calificaron como una tesis 
meritoria en la defensa doctoral en la Universidad del Valle.

De otra parte, para finalizar se analiza otro ejemplo en un rango más amplio (de centurias) sobre el desarrollo económico e institucional en Colombia (Ver figura 5):

Figura 5. Desarrollo sectorial en Colombia (Siglos XVI a XXI).

\begin{tabular}{c|ccc} 
Siglos XVI - XIX & Siglo XX & Siglo XIX \\
\cline { 2 - 4 } & Extractivismo en el sector prim ario & Industria- Sector Secundario & Ex tractivismo-Reprim arizacion
\end{tabular}

Fuente: Elaboración propia

Al analizar de manera intuitiva y visual la figura cinco se evidencia también claramente la presencia de path dependence. Hay un rango de tres tiempos asi: Uno, entre los siglos XVI al decimonónico donde los mayores réditos económicos se concentran en la explotación de metales en especial el oro y la plata para exportarlos al mercado internacional. En esta periodización se destaca que la actividad económica se desarrolló en el sector primario dado que la industria aparece muy tardíamente a final del siglo XIX. Dos, en siglo veinte el motor del desarrollo en el país se desempeñó y encumbro sobre la industria (siendo este sector el eje hegemónico más importante hasta su pauperización después de 1974). Y tres, el nuevo milenio devela que las nuevas apuestas del desarrollo económico en Colombia giran sobre los comodities, es decir la explotación de recursos naturales miniero-energeticos e hidrocarburos como: el petróleo, el gas y el carbón (denominados comodities). En suma, el desarrollo colombiano se presenta en tres tiempos: Uno, del siglo XVI al XIX bajo el extractivismo de oro y plata. Dos, en siglo veinte el sector de desarrollo más relevante es la industria. $Y$ tres, se presencia el retorno a actividades productivas del sector primario bajo el extractivismo de recursos naturales no renovables. Con este hecho se prueba una vez más una dependencia del pasado histórico (path dependence) de los sectores productivos en Colombia (Vergara, 2019).

Finalmente, en esencia el path dependence debe mostrar una trayectoria de camino en un contexto de larga duración histórica. Para analizar la dependencia de camino o path dependence se debe tener claro el modelo del institucionalismo histórico tal cual como se explicó aquí. Valga la pena recordar que en español no hay muy buenas traducciones de este modelo del institucionalismo histórico, esto explica en parte también porque no hay muchos estudios e investigaciones con aplicación de este marco teórico. En razón a esto este artículo busca socializar pedagógicamente al medio académico y al público en general esta herramienta de análisis para que a futuro se realicen otros estudios como este en el conjunto de las disciplinas que se inscriben en las ciencias sociales tanto en pregrado como en postgrado.

\section{BIBLIOGRAFÍA}

Basabe, S. (2007). "Instituciones e institucionalismo en América Latina". Editorial del Centro de Investigaciones Políticas y Económicas (CIPEC). Quito.

Boussaguet, Laurie. (2016). “Diccionario de políticas públicas". Publicación: Universidad Externado de Colombia. Bogotá. 
David, Paul A. (1994). Why are institutions the 'carriers of history'? Path dependence and the evolution of conventions, organizations and institutions. Structural change and economic dynamics. vol. 5, no 2, p. 205-220.

David, Paul A. (2001). "Path dependence, its critics and the quest for "historical economics". Evolution and path dependence in economic ideas: Past and present. vol. 15, p. 40. Oxford University. United Kingdom.

Fajardo, E. (2016). Hacia la caracterización de los valores democráticos y ciudadanos de las niñas y niños escolares: una mirada desde la filosofía para niños. Revista Amauta, 14(27), 71-86.

Farfán, G. (2007). “El nuevo institucionalismo histórico y las políticas sociales”. En: Revista Polis, vol. 3, \#1, p. $87-124$.

Fierro, B. (2015). Literatura e identidad, vasos comunicantes contra la desmemoria. Revista Amauta. Barranquilla (col.): Universidad del Atlántico Nro. 25, ene-Jun 2015.pp. 39-49 http://investigaciones.uniatlantico.edu.co/revistas/index.php/Amauta/article/view/1 283/911

García, Raimundo. (2013). “Del institucionalismo histórico al neo institucionalismo en la docencia e investigación en el caso mexicano". Instituto de Investigaciones Jurídicas de la UNAM. México.

Herrera Tapias, Beliña. (2013). "La constitucionalización de los derechos del consumidor en Colombia: un análisis desde los derechos sociales fundamentales". Civilizar ciencias sociales y humanas. Vol. 13, no 25 , p. 33-48.

Hidrón, Javier Henao. "Panorama del derecho constitucional colombiano". Temis, 2001.

Liebowitz, Stan J.; Margolis, Stephen E. (1995). “Path dependence, lock-in, and history”. JL Econ. \& Org. Vol. 11, p. 205.

Maldonado Copello, Alberto; Bonilla, Guido Alberto; Zamudio, José Vicente. "Efectos de la descentralización política en Colombia". Nómadas (Col), 2001, no 14, p. 272-275.

Marenco A. (2006). Path-Dependency, instituciones políticas y reformas electorales en perspectiva comparada. En: Revista Ciencia Política. Vol. 26. No 2. pp. 53- 75.

Marín - García, J.; Perello, M.; García S, J. (s.f.). “Desarrollo de una metodología para identificar dependencia de camino en gestión de operaciones". En: Working Papers on Operations Management, vol. 1, \#1, p. $37-40$.

Murillo, Guillermo.; González, Carlos.; García Mónica. (2010). "Cambio institucional y organizacional”. Cali: Editorial Universidad del Valle.

North, Douglass Cecil. (1990). "Institutions, institutional change and economic performance". Cambridge University Press.

North, Douglass Cecil. (1994). "Economic performance through time". The American economic review. Vol. 84, no 3, p. 359-368.

Olano, A. (2014). "El institucionalismo histórico comparado". Lecturas sobre América Latina. En: Revista Opera, \# 15, p. 5 - 29.

Paredes, Zioly; Díaz, Nordelia. (2007). “Los orígenes del Frente Nacional en Colombia”. Presente y Pasado. Revista de Historia. Vol. 12, no 23, p. 179-190.

Pening Gaviria, Jean Philippe. (2003). “Evaluación del proceso de descentralización en Colombia”. Economía y desarrollo, 2(1), 125.

Piña López, L. E., Medina Zárraga, N., \& Curiel Gutiérrez, I. (2017). Lectura crítica al manifiesto de Cartagena desde la perspectiva del liderazgo emergente. AMAUTA, 15(29), 65-73. https://doi.org/10.15648/ 
am.29.2017.5

Perdomo, Jaime Vidal. (2004). “Reforma administrativa de 1968: antecedentes, teoría de la organización y funcionamiento, sus principales consecuencias". Revista Vniversitas. No 108, p. 317-334.

Saavedra, S. (2016). “Análisis del desarrollo institucional desde el enfoque institucional histórico". En: Revista Papel Político, vol. 21, \#1. Bogotá: Pontificia Universidad Javeriana.

Sánchez De Dios, Manuel. (2004). “Estudio comparado de Path - dependence del Estado de bienestar en los casos de USA, Suecia, y España”. En: Revista de estudios políticos, abril - junio 2004, p. 95 - 128.

Sánchez, Miguel García. (2000). “Elección popular de alcaldes. El sistema de partidos en el ámbito municipal. 1988-1997". Análisis político. No 41, p. 84-98.

Trigo, L. (2015). El debate sobre la noción de Path Dependence y su conciliación en un modelo dinámico de análisis institucional. En: Revista chilena de derecho y ciencia política, Enero -Abril 2015 • ISSN 07189389 • e-ISSN 0719-2150 • Vol. 6 • № 1 Págs. 81-107.

Trigo, L. (2016). “Una revisión de los aportes del institucionalismo histórico a la ciencia política”. En: Revista Colombiana de Ciencias Sociales, vol. 7, \#1, p. $224-241$.

Valdivieso, Susana. (2008). "North y el cambio histórico: luces y sombras de la nueva historia institucional". Revista de economía institucional. Vol. 3, no 4. Bogotá.

Vergara Varela, Rafael. (2018). “Planes de desarrollo en Colombia y Management Publico. Un estudio bajo la técnica cualitativa del análisis de contenido". Revista Económicas CUC. Vol 39. No. 2.

Vergara Varela, Rafael. (2019). “Conformación histórico-institucional en Colombia (1800-1950)". Revista Justicia. Vol. 24 Núm. 36.

Vergara Varela, Rafael. (2019). “Management público y planes de desarrollo en Colombia (1958-2014). Un análisis a los sectores agropecuario e industrial”. Tesis Doctoral (Calificación Meritoria). Doctorado en Administración. Facultad de Ciencias de la Administración. Universidad del Valle. Cali, Colombia. 